\section{Rechnergestützte Regelung eines Prozesses zur simultanen Kohlenstoff- und Stickstoff-Elimination*}

\section{Bernd Striegel, Birgit Pellio, Dirk Weuster-Botz und Christian Wandrey*}

Im Bereich der Abwassertechnik gewinnt neben dem herkömmlichen CSB-Abbau die Stickstoffproblematik zunehmend an Bedeutung. Als Behandlungsmethode bietet sich neben Verfahren wie Knickpunkt-Chlorierung, Strippen oder Flüssigmembrantechnik [1] die biologische Aufarbeitung mittels Nitrifikation (N) und Denitrifikation (DN) an. Die Nitrifikation läuft in aerobem Milieu als Folgereaktion von Ammoniak über Nitrit zu Nitrat ab:

$$
\begin{aligned}
& \mathrm{NH}_{3}+1,5 \mathrm{O}_{2} \rightarrow \mathrm{NO}_{2}^{-}+\mathrm{H}_{2} \mathrm{O}+\mathrm{H}^{+}, \\
& \mathrm{NO}_{2}^{-}+0,5 \mathrm{O}_{2} \rightarrow \mathrm{NO}_{3}^{-} .
\end{aligned}
$$

Unter anoxischen Bedingungen kann das gebildete $\mathrm{NO}_{x}^{-}$als Elektronenakzeptor anstelle von Sauerstoff in der Denitrifikation zur Oxidation von kohlenstoffhaltigen Substraten genutzt werden:

$\mathrm{NO}_{3}^{-}+$org. C-Quelle $\rightarrow \mathrm{N}_{2}$

Aufbauend auf Arbeiten zur Modellierung und Charakterisierung von Reinkulturen für Nitrifikation und Denitrifikation werden die Möglichkeiten zur Reinigung komplexer, hochbelasteter Abwässer bei simultaner Kohlenstoff- und Stickstoff-Elimination untersucht. Den möglichen Verfahrensablauf zeigt eine bestehende großtechnische Anlage, in der seit über zehn Jahren Raffinerieabwässer in einer fünfstufigen Biologie behandelt werden [2].

\section{Zielsetzung}

Einen großen Teil der laufenden Kosten einer Abwasseranlage verursachen die Betriebsmittel Energie für $\mathrm{O}_{2}$-Eintrag, Säure- und Laugenverbrauch für $\mathrm{pH}$-Statisierung und Zudosieren einer externen C-Quelle für die nachgeschaltete Denitrifikation. Durch eine geeignete Prozeßführung sind diese Kosten unter Einhaltung aller geforderten Grenzwerte zu minimieren. Um den ProzeB kontrollieren zu können, ist eine ausreichende Information über den Prozeßzustand erforderlich. Dies wird mit Hilfe der KalmanFiltertechnik und einer geeigneten Meßausstattung angestrebt.

\section{Vorgehensweise}

Zum Erreichen einer vollständigen Elimination sowohl des Kohlenstoffs als auch des Stickstoffs ist das Verfahren der vorgeschalteten Denitrifikation sinnvoll. Dieses Konzept bietet den Vorteil, den vorhandenen CSB im Abwasser zur Elimination des aus der Nitrifikation rückgeführten $\mathrm{NO}_{\mathrm{x}}^{-}$einsetzen zu können. Durch gezieltes Einstellen des Rücklaufstroms wird zum einen in die Nitrifikation einzutragender Sauerstoff gespart, zum zweiten der

\footnotetext{
* Vortrag von B. Striegel auf dem Jahrestreffen der VerfahrensIngenieure, 25. bis 27. Sept. 1991 in Köln.

** Dipl.-Ing. B. Striegel, B. Pellio, Dr.-Ing. D. Weuster-Botz und Prof. Dr. C. Wandrey, Forschungszentrum Jülich GmbH, Institut für Biotechnologie, 5170 Jülich.
}

Bedarf an externer Kohlenstoffquelle für die nachgeschaltete Denitrifikation gesenkt.

Die Untersuchungen werden in zwei Fermentern (DN: 2,61, $\mathrm{N}$ : 14,31) mit je einem Sedimenter zur Biomasse-Rückhaltung $(1,4$ l) durchgeführt (Abb. 1). Zur Informationsgewinnung kommt ein semikontinuierlich arbeitendes erweitertes Kalman-Filter [3] zum Einsatz (Abb. 2).

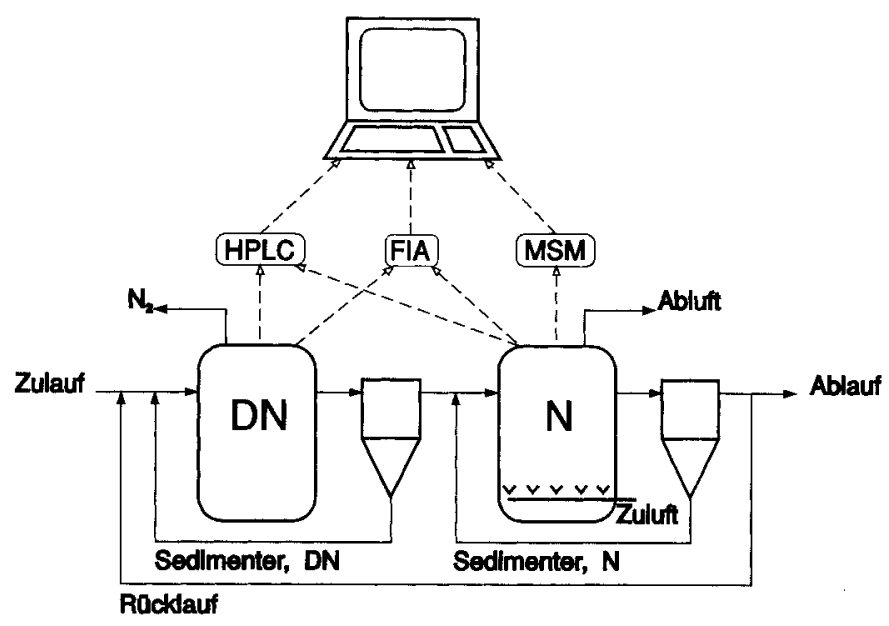

Abb. 1. Schema der Versuchsanlage (FIA = Fließ-InjektionsAnalytik, MSM = Massenspektrometer).

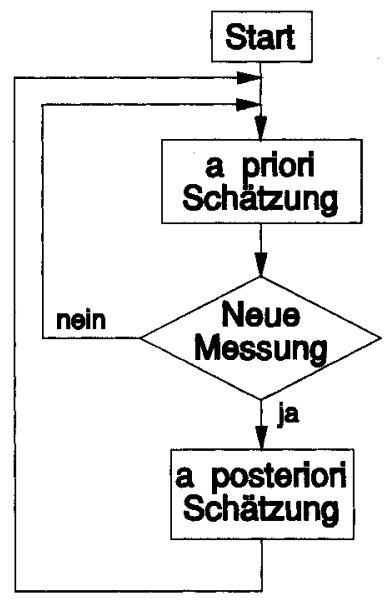

Abb. 2. Prinzip des semikontinuierlichen Kalman-Filters.

\section{Ergebnisse}

Der Rücklaufstrom ist ein wichtiger Parameter für die Gesamtabbauleistung einer Nitrifikation mit vorgeschalteter Denitrifikation (Abb. 3). Dabei wird die Abbauleistung für $\mathrm{NH}_{\mathrm{x}}$ oberhalb eines kritischen Volumenstroms durch Sedimenterüberlastung limitiert. Gleichzeitig verringert sich die Menge freigesetztes $\mathrm{NO}_{\mathrm{x}}^{-}$um bis zu $50 \%$. Der Umsatz von $\mathrm{NO}_{\mathrm{x}}^{-}$ist festgelegt als das Verhältnis von in der Nitrifikation produziertem zu in der Denitrifikation eliminiertem $\mathrm{NO}_{\mathrm{x}}^{-}$.

Aus Simulationsrechnungen für Biomasse, Wachstum und Konzentration resultiert, daß zur vollständigen Prozeßbeobachtung im Gegensatz zum einfachen Rührkessel ein aus Konzentrationsmessungen bestehender Meßvektor nicht ausreichend ist. Als zusätz- 


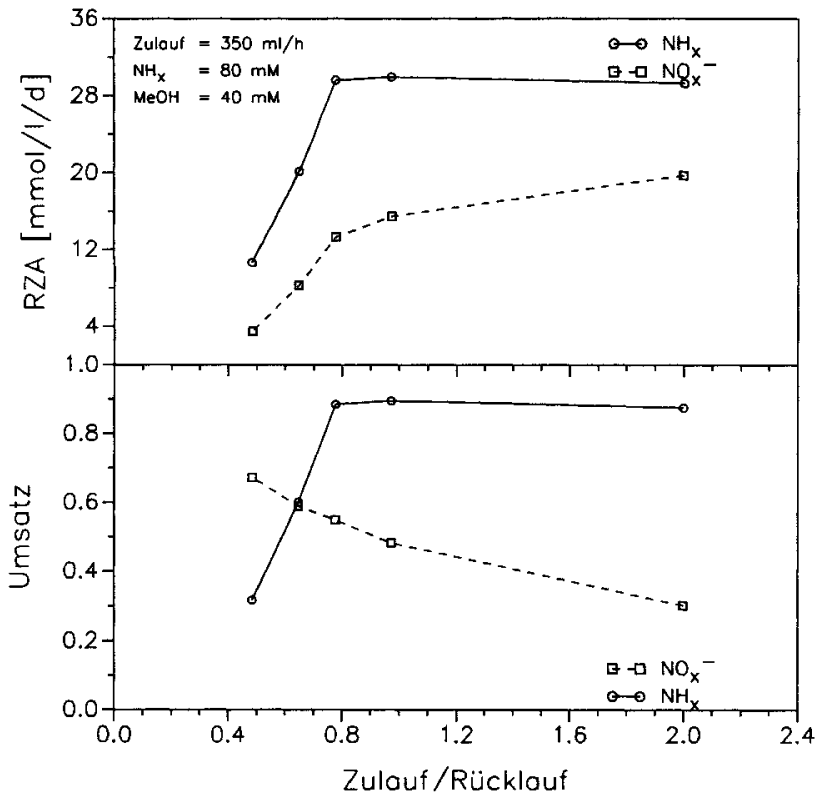

Abb. 3. Stationäre Betriebzustände bei verschiedenen Rücklaufströmen.

liche Information wird eine der Größen Biomasse, Wachstumsrate oder Sedimenterwirkungsgrad benötigt. Mit der Messung des Sedimenterwirkungsgrades alle $24 \mathrm{~h}$ ergibt sich bei einer angenommenen dynamischen Beaufschlagung (Sinusschwingung: Amplitude $20 \%$ vom Mittelwert, Periode $1 \mathrm{~d}$ ) der Anlage eine Mindestabtastfrequenz von etwa einer Konzentrationsmessung pro Stunde, unterhalb derer die dynamischen Verläufe nur schlecht nachvollzogen werden.

\section{Schlußfolgerungen}

Eine feste Einstellung des Rücklaufstroms bei Abwasseranlagen mit variabler Last ist nicht sinnvoll. Es besteht ein erhebliches Einsparungspotential hinsichtlich des einzutragenden Sauerstoffs und des zu eliminierenden Rest- $\mathrm{NO}_{\mathrm{x}}^{-}$.

Die erfolgreiche Umsetzung von Prozeßführungsstrategien hängt in starkem Maße davon ab, ob eine On-line-Analytik zur Verfügung steht, die mit ausreichender Abtastfrequenz arbeitet. Je höher der Informationsbedarf einer solchen Strategie ist, desto leistungsfähiger muß die zu installierende Analytik sein, insbesondere wenn mit nicht direkt meßbaren Größen gearbeitet wird, auf die erst nach Zwischenschalten eines Filter-Algorithmus zugegriffen werden kann.

Eingegangen am 27. November 1991

\section{Literatur}

[1] Dombrowski, T.; Schulz; W.; Wiesmann U.: Chemische Industrie (1988) Nr. 9, S. 91/94.

[2] Scholtyssek, H.: Dissertation, TU Clausthal 1976.

[3] Wiechert, W.: Dissertation, Universität Bonn 1991.

Schlüsselwörter: Nitrifikation, Denitrifikation, Kalman-Filter, Prozeßführung, Abwasser.

Das vollstăndige Manuskript der Arbeit umfaßt 17 Seiten mit 12 Abbildungen, 2 Tabellen und 8 Literaturzitaten. Es ist als Fotokopie oder Mikrofiche MS 2066/1992 erhältlich. 